\title{
Multipactor susceptibility on a dielectric with a bias dc electric field and a background gas
}

Peng Zhang, Y. Y. Lau, Matthew Franzi, and R. M. Gilgenbach

Citation: Phys. Plasmas 18, 053508 (2011); doi: 10.1063/1.3592990

View online: http://dx.doi.org/10.1063/1.3592990

View Table of Contents: http://pop.aip.org/resource/1/PHPAEN/v18/i5

Published by the AIP Publishing LLC.

\section{Additional information on Phys. Plasmas}

Journal Homepage: http://pop.aip.org/

Journal Information: http://pop.aip.org/about/about_the_journal

Top downloads: http://pop.aip.org/features/most_downloaded

Information for Authors: http://pop.aip.org/authors

\section{ADVERTISEMENT}

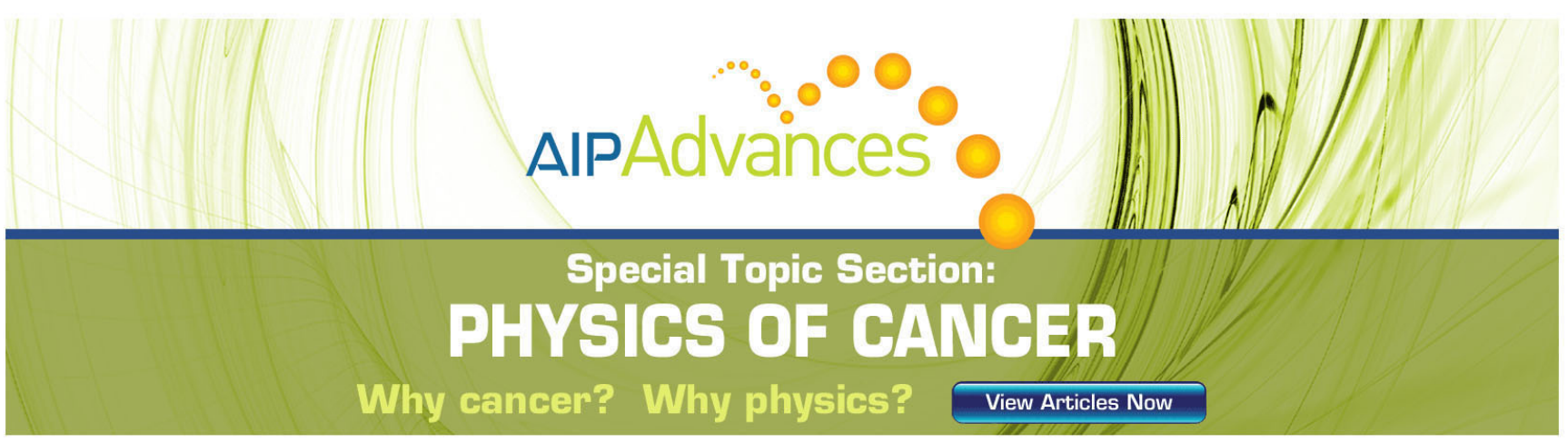




\title{
Multipactor susceptibility on a dielectric with a bias dc electric field and a background gas
}

\author{
Peng Zhang, Y. Y. Lau, ${ }^{a)}$ Matthew Franzi, and R. M. Gilgenbach \\ Department of Nuclear Engineering and Radiological Sciences, University of Michigan, Ann Arbor, \\ Michigan 48109-2104, USA
}

(Received 17 March 2011; accepted 2 May 2011; published online 31 May 2011)

We use Monte Carlo simulations and analytical calculations to derive the condition for the onset of multipactor discharge on a dielectric surface at various combinations of the bias dc electric field, $\mathrm{rf}$ electric field, and background pressures of noble gases, such as Argon. It is found that the presence of a tangential bias dc electric field on the dielectric surface lowers the magnitude of $\mathrm{rf}$ electric field threshold to initiate multipactor, therefore plausibly offering robust protection against high power microwaves. The presence of low pressure gases may lead to a lower multipactor saturation level, however. The combined effects of tangential dc electric field and external gases on multipactor susceptibility are presented. (C) 2011 American Institute of Physics. [doi:10.1063/1.3592990]

\section{INTRODUCTION}

Multipactor discharge is an ubiquitous phenomenon observed in a multitude of devices that employ microwaves. ${ }^{1}$ It may occur when a metallic gap or a dielectric surface is exposed to an ac electric field under some favorable conditions, and its avoidance has been a major concern among workers on high power microwave (HPM) sources, rf accelerators, and space-based communication systems. ${ }^{1-15}$

$\mathrm{RF}$ window breakdown or dielectric failure ${ }^{2-11}$ has been a limiting factor in many high power microwave systems. In general, an avalanche of secondary electrons caused by multipactor discharge occurs in the prebreakdown phase. When this avalanche of electrons reaches a sufficiently high saturation level, it induces appreciable outgassing from the dielectric surface. Further ionization caused by these electrons provides a gaseous-like discharge (sometimes called flashover) within the desorbed gas layer, and it eventually turns into the breakdown phase of the dielectric. ${ }^{7-9}$ Due to the high susceptibility of multipactor discharge on dielectric, ${ }^{4-6}$ a small amount of seed electrons may grow to a high level that will initiate the breakdown of dielectric. To prevent the breakdown, it is necessary to suppress or eliminate the initial multipactor discharge.

In this paper, we extend the idea of the transmitter-receiver (T-R) switch typically used in radar systems, which offers automatic protective isolation to electronic circuits during its operation. During the operation of the transmitter in radar systems, the vacuum gap across the receiver is caused to spark by a T-R vacuum tube (typically filled with argon and water vapor at reduced pressure), so that the effect is that of placing a short-circuit across the receiver, the latter will be protected from a large influx of energy. ${ }^{16}$ When a dielectric window is exposed to HPM sources, we seek to utilize multipactor initiation on the dielectric surface so that the window becomes lossy and unmatched. Thus, the pres-

\footnotetext{
a)Electronic mail: yylau@umich.edu.
}

ence of HPM may induce surface multipactor discharge that blocks further propagation of the HPM, preferably at a low power level of HPM.

The theory of multipactor discharge on a dielectric surface has been extensively researched, including Monte Carlo (MC) particle simulations, ${ }^{4}$ dynamic theory, ${ }^{5}$ particle-in-cell (PIC) simulations, ${ }^{17,18}$ analytical calculations, ${ }^{4}$ and statistical theory. ${ }^{10}$ A typical susceptibility diagram for multipactor discharge is shown in Fig. 1, indicating the lower and upper boundaries of the rf electric field within which multipactor may occur. ${ }^{4}$ Roughly, the lower (upper) boundary corresponds to electron impact energy on the dielectric surface equal to the first (second) crossover point in the secondary electron yield curve $\mathrm{e}^{4}$ and multipactor saturation occurs at the lower boundary. ${ }^{5}$ The effects of space charge, ${ }^{19}$ external magnetic field, ${ }^{20,21}$ oblique rf electric fields, ${ }^{20}$ wave reflection, ${ }^{21}$ desorption gases, ${ }^{22}$ and external perpendicular dc bias electric field ${ }^{23}$ on multipactor discharge on a dielectric have been investigated. The transition of window breakdown from vacuum multipactor discharge to rf plasma has also been studied, by both PIC simulations ${ }^{17,18}$ and volume-averaged global model $(\mathrm{GM}){ }^{24,25}$ Simple analytical scaling laws for dielectric window breakdown in vacuum and collisional regimes have been derived. ${ }^{26}$

To initiate multipactor discharge at a lower power level of HPM, we introduce a bias-dc electric field that is parallel to the dielectric surface. This additional dc electric field is expected to lower the threshold of the rf electric field for multipactor initiation. That a tangential dc electric field may reduce the $\mathrm{rf}$ threshold is supported by the well-known limit of dc dielectric breakdown. ${ }^{9,15,27-32}$ Here, we quantify the calculations. We use MC simulations and analytical calculations to derive the condition for the onset of multipactor discharge at various combinations of the bias dc electric field, rf electric field, and background pressures of some noble gases, such as Argon.

To understand the individual effects of the tangential dc bias electric field and of the low pressure background gas, in 


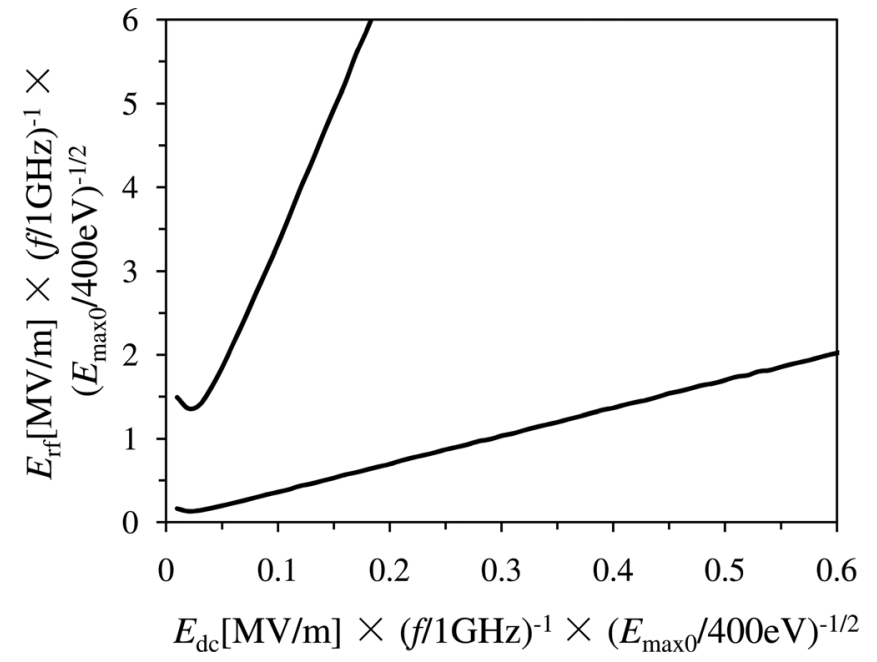

FIG. 1. Multipactor susceptibility boundaries from Monte Carlo simulation, in the $\left(E_{\mathrm{dc}}, E_{\mathrm{rfO}}\right)$ plane for $\delta_{\max 0}=3$, and $E_{\mathrm{om}} / E_{\max 0}=0.005$. Here, $E_{\mathrm{rf} 0}$ is the amplitude of the tangential rf electric field at frequency $f, E_{\mathrm{dc}}$ is the charging electric field on the dielectric surface, $\delta_{\max 0}$ is the maximum secondary electron yield occuring at impact energy $E_{\max 0}$, and $2 E_{\text {om }}$ is the average emission energy of secondary electrons.

Sec. II, we first consider the effect of a dc electric field tangential to the dielectric surface. In this case, the electrons will gain energy from both rf electric field and the tangential dc electric field during the time of flight, leading to a lower threshold for the rf electric field for multipactor initiation. In Sec. III, we remove the tangential electric field but introduce a low-pressure background gas. Under such conditions, the collisions between electrons in flight with the background neutral gas molecules have to be taken into consideration. Lastly, the combined effects of the tangential dc electric field and the presences of gas background will be presented in Sec. IV. Concluding remarks will be given in Sec. V.

\section{EFFECT OF TANGENTIAL DC ELECTRIC FIELD}

First, we consider the effect of only a tangential dc electric field. The multipactor electrons are acted on by the $\mathrm{rf}$ electric field $E_{r f} \sin (\omega t+\theta)$ and tangential dc electric field $E_{t}$, and by the charging electric field $E_{d c}$. Here, $E_{d c}$ is assumed constant along the $x$-direction (Fig. 2), where the possible space-charge effects due to multipactor electrons ${ }^{17,19}$ are not considered. Referring to Fig. 2, from the force law

$$
m \frac{\partial \vec{v}}{\partial t}=-|e|\left[\vec{E}_{r f} \sin (\omega t+\theta)+\vec{E}_{t}+\vec{E}_{d c}\right]
$$

we obtain

$$
\begin{gathered}
v_{x}=-\frac{|e|}{m} E_{d c} t+v_{o} \sin \phi, \\
v_{y}=\frac{|e|}{m \omega} E_{r f}[\cos (\omega t+\theta)-\cos \theta]-\frac{|e|}{m} E_{t} t+v_{o} \cos \phi,
\end{gathered}
$$

where the last terms account for the emission velocity at $t=0$. From Eq. (2), it is clear that the transit time is given by

$$
\tau=2 m v_{o} \sin \phi /|e| E_{d c}
$$

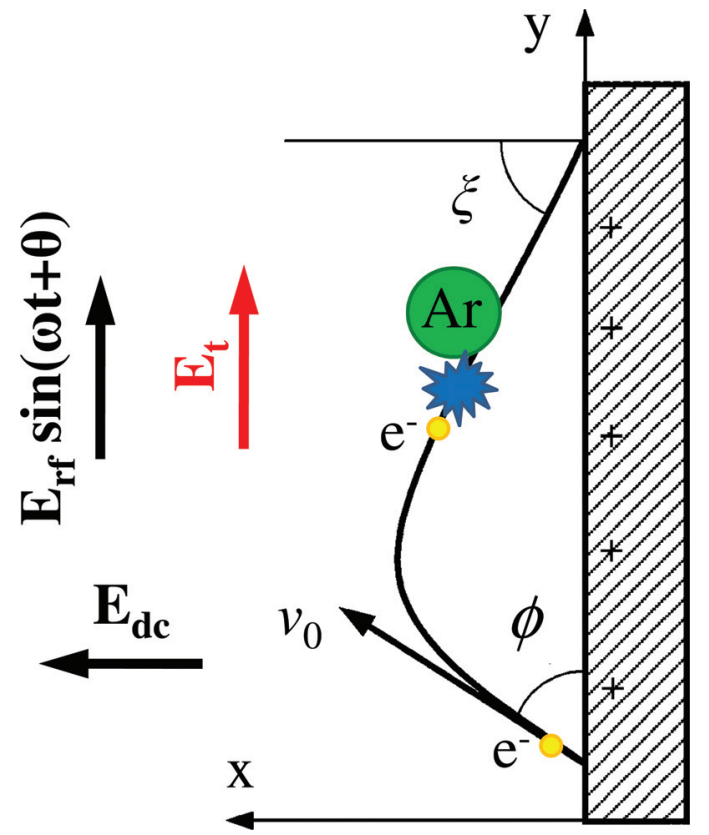

FIG. 2. (Color online) Schematic of a single-surface multipactor in a parallel rf and normal dc electric fields, with the presence of an external tangential dc electric field, and (or) low pressure gases.

Upon impact on the surface, a primary electron produces an average number of secondary electrons, called the secondary electron yield, $\delta$. This yield depends on the material and is a function of the impact energy of the primary electron, $E_{\mathrm{i}}$, and the angle to the normal, $\xi$, at which it strikes the surface. ${ }^{33}$ For the dependence of yield on impact energy $E_{\mathrm{i}}$, we will adopt Vaughan's empirical formula, ${ }^{33}$ for normal incidence

$$
\delta=\delta\left(E_{i}\right) \cong \delta_{\max }\left(w e^{1-w}\right)^{k},
$$

where $\delta_{\max }$ is the maximum value of $\delta, w=E_{i} / E_{\max }, E_{\max }$ being the impact energy which yields $\delta_{\max }$, and $k=0.62$ for $w<1$ and $k=0.25$ for $w=1$. Two values of impact energy, termed the first and second crossover points, $E_{1}$ and $E_{2}$, respectively, result in a yield of 1 , while $\delta>1$ in between. For impact at an angle $\xi$ with respect to the normal (Fig. 2), the parameters are adjusted in calculating the yield, according to the following equations ${ }^{33}$ :

$$
\begin{aligned}
& E_{\max }=E_{\max 0}\left(1+\frac{k_{s} \xi^{2}}{\pi}\right) \\
& \delta_{\max }=\delta_{\max 0}\left(1+\frac{k_{s} \xi^{2}}{2 \pi}\right) .
\end{aligned}
$$

Here $E_{\max 0}$ and $\delta_{\max 0}$ are the parameters for an impact angle $\xi=0$ (i.e., normal to the surface), and $k_{\mathrm{s}}$ is a surface smoothness factor ranging from 0 for a rough surface to 2 for a polished surface. In this paper we set $k_{\mathrm{s}}=1$, representing a typical dull surface. ${ }^{33}$ It is worth noting that in this situation, since the electrons gain their energy from the parallel electric field, most impacts will be at almost grazing incidence (i.e., $\xi \cong \pi / 2$ ). 
To calculate the evolution of the multipactor discharge, we follow the trajectory of a weighted macroparticle over a large number of impacts in a MC simulation. ${ }^{4,5}$ The initial $\mathrm{rf}$ phase, $\theta$, is uniformly distributed over $0<\theta<2 \pi$ (Fig. 2). Each time a macroparticle leaves the surface, we assign it a random initial energy $E_{0}=\frac{1}{2} m v_{0}^{2}$ and angle $\phi$ according to the following distributions ${ }^{4}$ :

$$
\begin{gathered}
f\left(E_{0}\right)=\frac{E_{0}}{E_{o m}^{2}} e^{-\left(E_{0} / E_{o m}\right)}, \\
g(\phi)=\frac{1}{2} \sin \phi
\end{gathered}
$$

where $E_{\mathrm{om}}$ is the peak of the distribution of emission energies, on the order of the work function, i.e., a few eV.,.$^{1,4,5}$ Note that the expected value of $E_{\mathrm{o}}$ is $2 E_{\mathrm{om}}$, and that $\int g(\phi) d \phi=1$ over $0<\phi<\pi$. Substituting the random values of initial velocity (energy) and angle into Eqs. (2) and (3), we obtain the impact energy and impact angle, hence, the secondary electron yield from Eq. (5) after time of flight $\tau$ that is given by Eq. (4). We use this value of the yield to adjust the charge on the macroparticle and then emit it again with a random velocity and angle. We repeat the process to obtain a series of yield $\left(\delta_{1}, \delta_{2}, \ldots, \delta_{N}\right)$ for a large number of impacts. The average value of secondary yield over $N$ impacts is calculated as $\bar{\delta}=\left(\delta_{1} \cdot \delta_{2} \cdot \ldots \delta_{N}\right)^{1 / N}{ }^{13}$ where $N=200$ is used in the calculation. In this fashion, we can determine either an exponentially growing $(\bar{\delta}>1)$ or an exponentially decaying $(\bar{\delta}<1)$ trend in the number of electrons in the avalanche, depending on the external parameters, such as $E_{\mathrm{dc}}, E_{\mathrm{rf} 0}, E_{\mathrm{t}}$, and $\delta_{\max 0}$. For any given values of the fields, the growth rate is determined by the average value of the secondary electron yield, averaged over the distributions of random emission energy, random emission angle, and random rf phase at emission. The boundaries of the multipactor susceptibility are determined when the exponential growth rate of the electrons equals zero.

Figure 3 shows the susceptibility diagram for the multipactor boundaries with the presence of tangential dc electric field. It is clear that the presence of a tangential dc electric field will significantly lower the magnitude of rf electric field required to initiate multipactor (as compared to Fig. 1), thus increasing the "area" of the multipactor susceptibility in the $\left(E_{\mathrm{rf}}, E_{\mathrm{dc}}\right)$ plane in which multipactor would occur. ${ }^{4,5}$ With the presence of tangential dc electric field, for example, $E_{\mathrm{t}}[\mathrm{MV} / \mathrm{m}] \times(f / 1 \mathrm{GHz})^{-1} \times\left(E_{\max 0} / 400 \mathrm{eV}\right)^{-1 / 2}=0.5$ in Fig. 3 (b), multipactor could readily occur in the low charging dc electric field regime, $E_{\mathrm{dc}}[\mathrm{MV} / \mathrm{m}] \times(f / 1 \mathrm{GHz})^{-1} \times\left(E_{\max 0} /\right.$ $400 \mathrm{eV})^{-1 / 2}<0.25$, even before the presence of if electric field. This roughly corresponds to the dc electric field threshold for electron avalanche on an insulator. ${ }^{29,30,32}$ However, the upper boundary of the multipactor is not sensitive to the tangential dc electric field, as shown in Fig. 3(a).

In order to construct simple analytic solutions for the susceptibility diagrams, we follow Ref. 4 and assume that all electrons are emitted normal to the surface (i.e., $\phi=90^{\circ}$ in Fig. 2), with a single energy $E_{0}=(1 / 2) m v_{0}^{2}$. Hence, substituting $\phi=90^{\circ}$ into Eqs. (2)-(4), averaging over rf phase $\theta$, and setting the resulting average impact energy equal to $E_{1}$
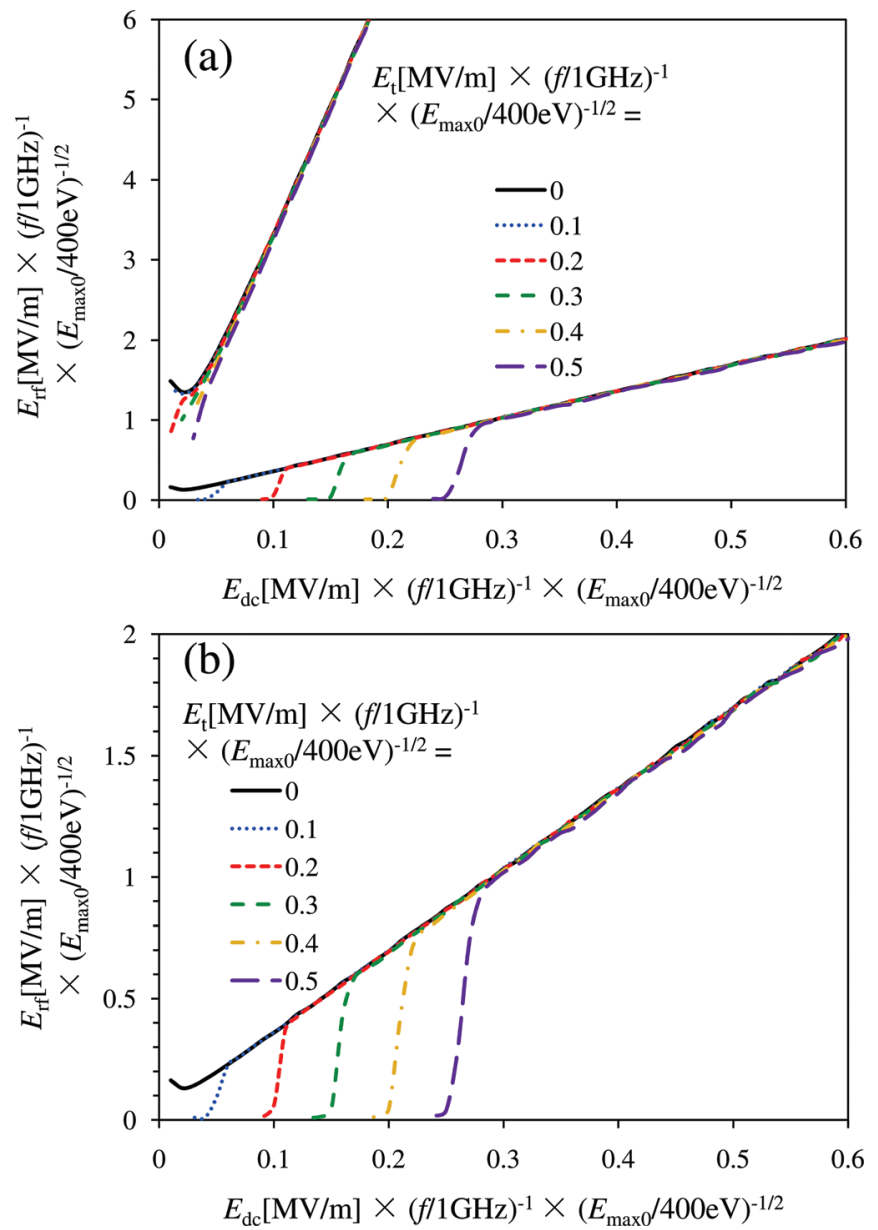

FIG. 3. (Color online) Multipactor susceptibility boundaries with various $E_{\mathrm{t}}$, from Monte Carlo simulation, in the $\left(E_{\mathrm{dc}}, E_{\mathrm{rf0}}\right)$ plane for $\delta_{\max 0}=3$, and $E_{\mathrm{om}} / E_{\max 0}=0.005$, (a) both upper and lower boundaries, (b) zoom in view of lower boundaries in (a).

and $E_{2}$ at $\xi \cong \pi / 2$ [cf., Eqs. (5) and (6)], we obtain the following equations for the lower and upper boundaries:

$$
\frac{|e| E_{r f}}{\omega \sqrt{m E_{\max o}}}=\sqrt{\frac{2 E_{1,2} / E_{\max o}-8\left(E_{t} / E_{d c}\right)^{2} E_{o} / E_{\max o}}{1-\cos \left(2 \omega \sqrt{2 m E_{o}} /|e| E_{d c}\right)}},
$$

which can be expressed in the normalized from as

$$
\bar{E}_{r f}=\sqrt{\frac{2 \bar{E}_{1,2}-8\left(\bar{E}_{t} / \bar{E}_{d c}\right)^{2} \bar{E}_{o}}{1-\cos \left(2 \sqrt{2 \bar{E}_{o}} / \bar{E}_{d c}\right)}},
$$

where $\quad \bar{E}_{r f}=\frac{|e| E_{r f}}{\omega \sqrt{m E_{\max }}}, \quad \bar{E}_{t}=\frac{|e| E_{t}}{\omega \sqrt{m E_{\max }}}, \quad \bar{E}_{d c}=\frac{|e| E_{d c}}{\omega \sqrt{m E_{\max }}}, \quad \bar{E}_{1,2}$ $=E_{1,2} / E_{\max o}$, and $\bar{E}_{0}=E_{0} / E_{\max o}$.

Figure 4 shows the multipactor region boundaries calculated from Eq. (10). The drastic simplification introduced to derive Eqs. (9) and (10) does not qualitatively change the solution shown in Fig. 3. Since the second cross-over impact energy $E_{2}$ is much higher than the emission energy $E_{0}$, adding a tangential dc electric field will not affect the upper boundaries of the susceptibility diagram much, also shown in Figs. 3(a) and 4(a). The slopes of the curves in Fig. (4), in the limit of large $E_{\mathrm{dc}}$, are $\sqrt{E_{1,2} / 2 E_{0}}$, as easily deduced from Eq. (10). By setting Eq. (9) equal to 0 , we obtain 

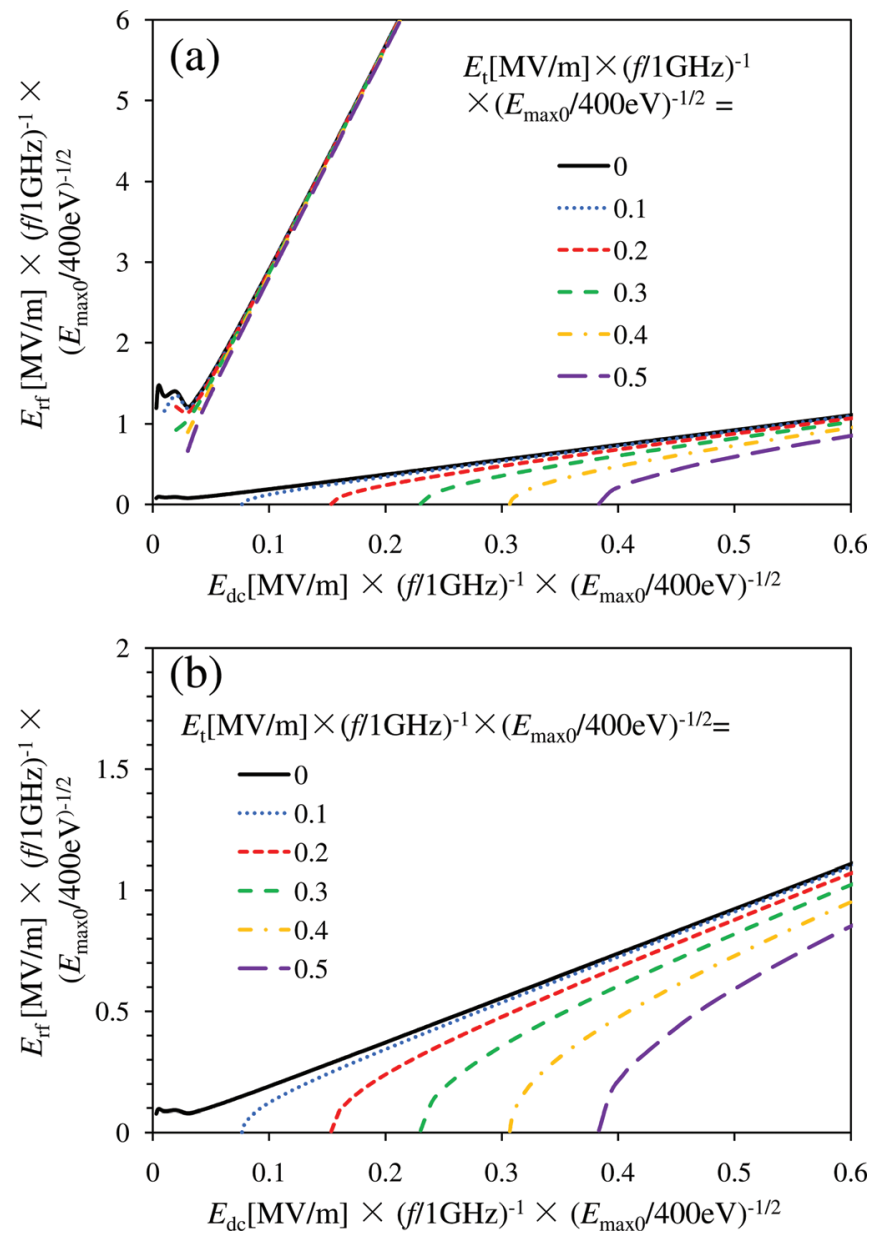

FIG. 4. (Color online) Multipactor susceptibility boundaries with various $E_{\mathrm{t}}$, from direct calculation of Eq. (10), in the $\left(E_{\mathrm{dc}}, E_{\mathrm{rfo}}\right)$ plane for $\delta_{\max 0}=3$, and $E_{\mathrm{om}} / E_{\max 0}=0.005$, (a) both upper and lower boundaries, (b) zoom in view of lower boundaries in (a).

$$
E_{d c}=2 E_{t} \sqrt{\frac{E_{0}}{E_{1}}}
$$

which is the maximum charging field below which multipactor would be possible even without an rf electric field, for a given tangential dc field $E_{\mathrm{t}}$, as shown in Figs. 3(b) and 4(b). Note that Eq. (11) is also the same expression for dc electric field threshold for electron avalanche on an insulator (cf., Eq. (A2) of Ref. 32).

\section{EFFECT OF LOW PRESSURE GAS}

With the presence of gases, the electrons emitted from the dielectric surface will undergo collisions with the gas molecules, before impacting the dielectric surface again, as schematically illustrated in Fig. 2. For simplicity, in this section we remove the tangential dc bias electric field and include the effect of low pressure gas only. In this case, the conservation of momentum ${ }^{34}$ gives

$$
m n_{e} \frac{\partial \vec{v}}{\partial t}=-|e| n_{e}\left[\vec{E}_{r f} \sin (\omega t+\theta)+\vec{E}_{d c}\right]-n_{e} m v_{m} \vec{v}-m \vec{v} G
$$

where $\vec{v}$ is the mean electron velocity, $n_{e}$ is the electron number density, $v_{m}$ is the momentum transfer collision frequency, $G=n_{e} v_{i}$ is the electron generation rate from ionization, and $v_{i}$ is the ionization frequency. By writing Eq. (12), we consider only the regime of low gas pressure, where the diffusion loss and other collisions, such as excitation and recombination, can be neglected. ${ }^{22}$

In Ref. 22, only the analytic solution to this problem was constructed under similar simplifying assumptions as in Ref. 4. Here we provide the MC simulation including a test on the sensitivity of the electron energy distribution functions used.

From Eq. (12) we have

$$
\begin{gathered}
\frac{\partial v_{x}}{\partial t}+v_{t} v_{x}=-\frac{|e| E_{d c}}{m}, \\
\frac{\partial v_{y}}{\partial t}+v_{t} v_{y}=-\frac{|e| E_{r f} \sin (\omega t+\theta)}{m},
\end{gathered}
$$

where $v_{t}=v_{i}+v_{m}$. Solving Eqs. (13) and (14), we have

$$
\begin{gathered}
v_{x}=-\frac{|e| E_{d c}}{m v_{t}}+\mathrm{e}^{-v_{t} t}\left(v_{0} \sin \phi+\frac{|e| E_{d c}}{m v_{t}}\right), \\
v_{y}=e^{-v_{t} t} v_{0} \cos \phi+\frac{|e| E_{r f} e^{-v_{t} t}}{m\left(\omega^{2}+v_{t}^{2}\right)}\left(v_{t} \sin \theta-\omega \cos \theta\right) \\
-\frac{|e| E_{r f}}{m\left(\omega^{2}+v_{t}^{2}\right)}\left[v_{t} \sin (\omega t+\theta)-\omega \cos (\omega t+\theta)\right]
\end{gathered}
$$

where $v_{0}$ is the initial emission velocity. The transit time, $\tau$, can be obtained from the condition $\int_{0}^{\tau} v_{x} d t=0$ as

$$
\left(1-\mathrm{e}^{-v_{t} \tau}\right)\left(v_{0} \sin \phi+\frac{|e| E_{d c}}{m v_{t}}\right)-\frac{|e| E_{d c}}{m} \tau=0 .
$$

To obtain the gas parameters $v_{m}$ and $v_{i}$, the electron-neutral momentum transfer collision cross sections $\sigma_{m}$ and ionization cross sections $\sigma_{i}$ need to be examined. In this study, we choose Argon as an example for simplicity in its chemistry; its ionization and other collision cross sections are well characterized $^{35-37}$ and straightforward (compared with air). At low gas pressure ( $<50$ Torr), the electron energy distribution may be approximated as Maxwellian, ${ }^{24}$

$$
f(\varepsilon)=\frac{2}{\sqrt{\pi}}\left(K_{B} T_{e}\right)^{-3 / 2} \varepsilon^{1 / 2} \exp \left(-\frac{\varepsilon}{K_{B} T_{e}}\right),
$$

where $K_{\mathrm{B}}$ is the Boltzmann's constant and $T_{\mathrm{e}}$ is the electron temperature. Thus, the collision frequencies $v_{m}$ and $v_{i}$ may be averaged over all electron energies as

$$
v_{m, i}=n_{g} \int_{0}^{\infty} f(\varepsilon) \sigma_{m, i}(\varepsilon) \sqrt{\frac{2 e \varepsilon}{m}} d \varepsilon,
$$

where $n_{\mathrm{g}}$ is the gas density. Dividing both sides of Eq. (19) by the gas pressure $p=n_{\mathrm{g}} K_{\mathrm{B}} T_{\mathrm{g}}$, we have

$$
\frac{v_{m, i}}{p}=\frac{1}{K_{B} T_{g}} \int_{0}^{\infty} f(\varepsilon) \sigma_{m, i}(\varepsilon) \sqrt{\frac{2 e \varepsilon}{m}} d \varepsilon,
$$




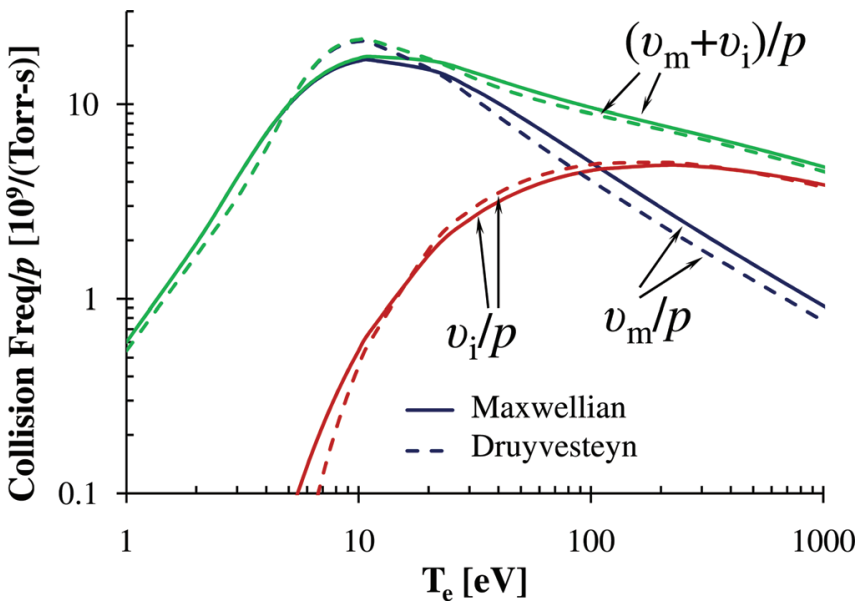

FIG. 5. (Color online) Momentum transfer collision $v_{\mathrm{m}} / p$ frequency and ionization frequency $v_{\mathrm{i}} / p$ for Argon as a function of electron temperature, according to Maxwellian and Druyvesteyn distributions.

where $T_{\mathrm{g}}\left(\ll T_{\mathrm{e}}\right)$ is the gas temperature, which is assumed to be the room temperature $(0.026 \mathrm{eV})$ in this study. By using the data in Refs. 35-37 for the ionization cross section $\sigma_{\mathrm{i}}$ and the electron-neutral momentum transfer collision cross section $\sigma_{\mathrm{m}}$ for Argon, Eq. (20) is numerically integrated. The results are shown in Fig. 5. The calculation is repeated for a non-Maxwellian electron energy distribution, namely, the Druyvesteyn distribution, ${ }^{24}$

$$
\begin{gathered}
f(\varepsilon)=A\left(K_{B} T_{e}\right)^{-3 / 2} \varepsilon^{1 / 2} \exp \left[-B\left(\frac{\varepsilon}{K_{B} T_{e}}\right)^{2}\right], \\
A=\frac{4[\Gamma(1 / 4)]^{4}}{\pi(12 \sqrt{2} \pi)^{3 / 2}}, \quad B=\frac{[\Gamma(1 / 4)]^{4}}{72 \pi^{2}},
\end{gathered}
$$

where $\Gamma$ is the gamma function. The results are also shown in Fig. 5. It is clear that the collision frequencies $v_{m}$ and $v_{i}$ are insensitive to the actual electron density distribution function.

In the low pressure regime, a multipacting electron experiences primarily the vacuum rf electric field during its time of flight $\tau$, from its birth on the dielectric surface as a secondary electron to its impact onto the dielectric surface as the primary electron for the next generation of the secondary electrons, after its acceleration by the vacuum rf electric field during $\tau$. For pressures less than 1 Torr, this condition is largely satisfied. In such cases, the multipacting electron typically has an energy on the order of $100 \mathrm{eV}$ to $1 \mathrm{keV},{ }^{6,38}$ for $\mathrm{rf}$ electric field of order of $1 \mathrm{MV} / \mathrm{m}$ and $\mathrm{rf}$ frequency below $10 \mathrm{GHz}$, regardless of the dielectric material. For electron energy on the order of $100 \mathrm{eV}$ to $1 \mathrm{keV}$, the composite value of $v_{t} / p=\left(v_{i}+v_{m}\right) / p$ changes little $\left(\sim 10^{10} \operatorname{Torr}^{-1} \mathrm{~s}^{-1}\right)$, perhaps by a factor of 2 at the most, as shown in Fig. 5. Thus, for simplicity, we use $v_{t} / p \approx 10^{10} \mathrm{Torr}^{-1} s^{-1}$ in subsequent calculations. Note that similar approximations are taken in Ref. 22 for Nitrogen.

Similar to Sec. II, to calculate the evolution of the multipactor discharge, we follow the trajectory of a weighted macroparticle over a large number of impacts in a MC simulation. The initial rf phase is uniformly distributed over $0<\theta<2 \pi$. Each time a macroparticle leaves the surface, we assign it a random initial energy $E_{0}=\frac{1}{2} m v_{0}^{2}$ and angle $\phi$ according to Eqs. (7) and (8). Substituting the random values of initial velocity (energy) and angle into Eqs. (15) and (16), we obtain the impact energy and impact angle, hence, the secondary electron yield from Eq. (5) after a transit time $\tau$ according to Eq. (17). We use this value of the yield to adjust the charge on the macroparticle and then emit it again with a random velocity and angle. We can determine either an exponentially growing or an exponentially decaying trend in the number of electrons in the avalanche, depending on the external parameters, such as $E_{\mathrm{dc}}, E_{\mathrm{rf} 0}, p$, and $\delta_{\max 0}$. For any given values of the fields, the growth rate is determined by the average value of the secondary electron yield, averaged over the distributions of random emission energy, random emission angle, and random RF phase at emission. The boundaries of the multipactor region are determined when the exponential growth rate of the electrons equals to zero., Here, we examine the averaged secondary electron yield from multipactor only; the electrons produced from ionization are not assigned to the "macroparticle" during its flight.

Figure 6 shows the effect of various gas pressures to the multipactor initiation. For a given normal dc electric field, the presence of gases will introduce a drag force to the
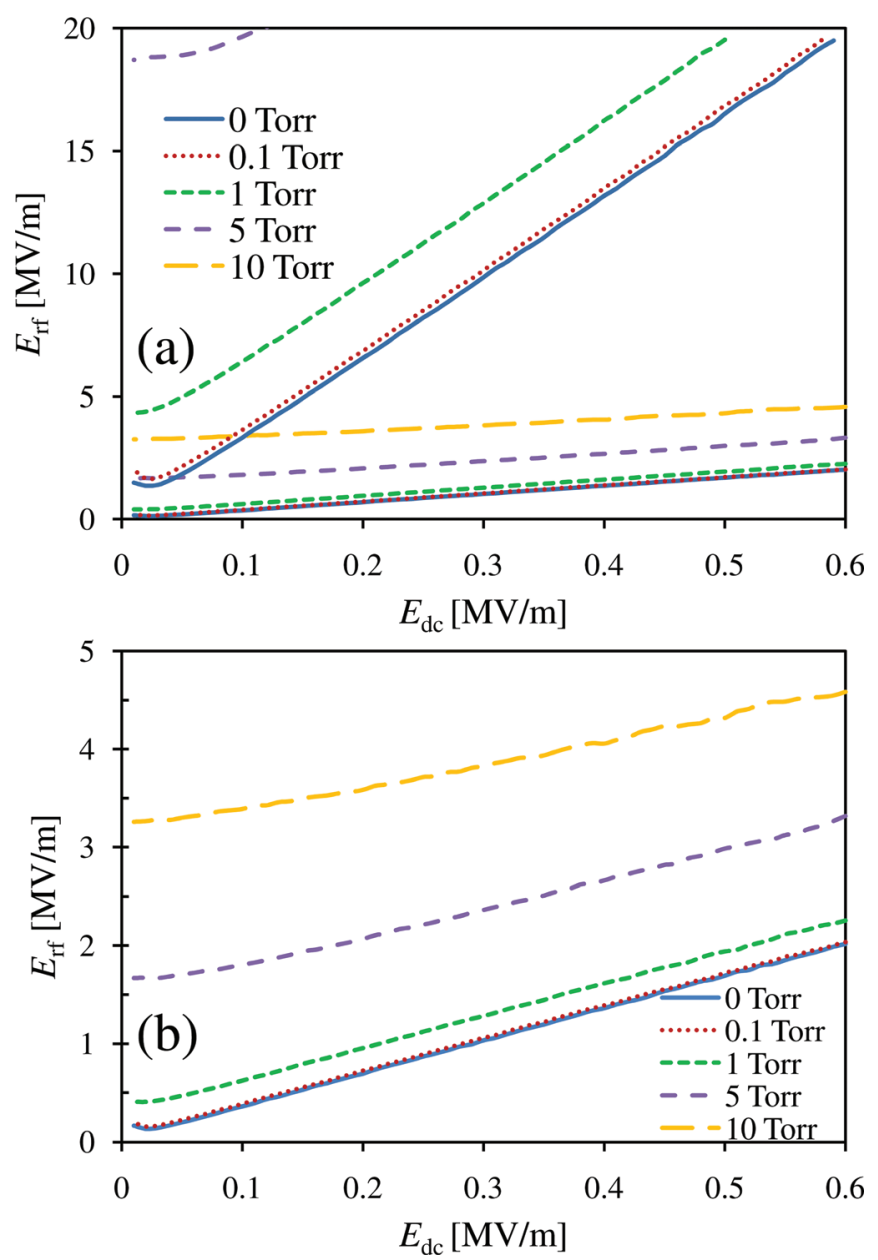

FIG. 6. (Color online) Multipactor susceptibility boundaries with gases, from Monte Carlo simulation, in the $\left(E_{\mathrm{dc}}, E_{\mathrm{rf0}}\right)$ plane for $\delta_{\max 0}=3$, $E_{\max 0}=400 \mathrm{eV}, f=1 \mathrm{GHz}$, and $E_{\mathrm{om}} / E_{\max 0}=0.005$, (a) both upper and lower boundaries, (b) zoom in view of lower boundaries in (a). 

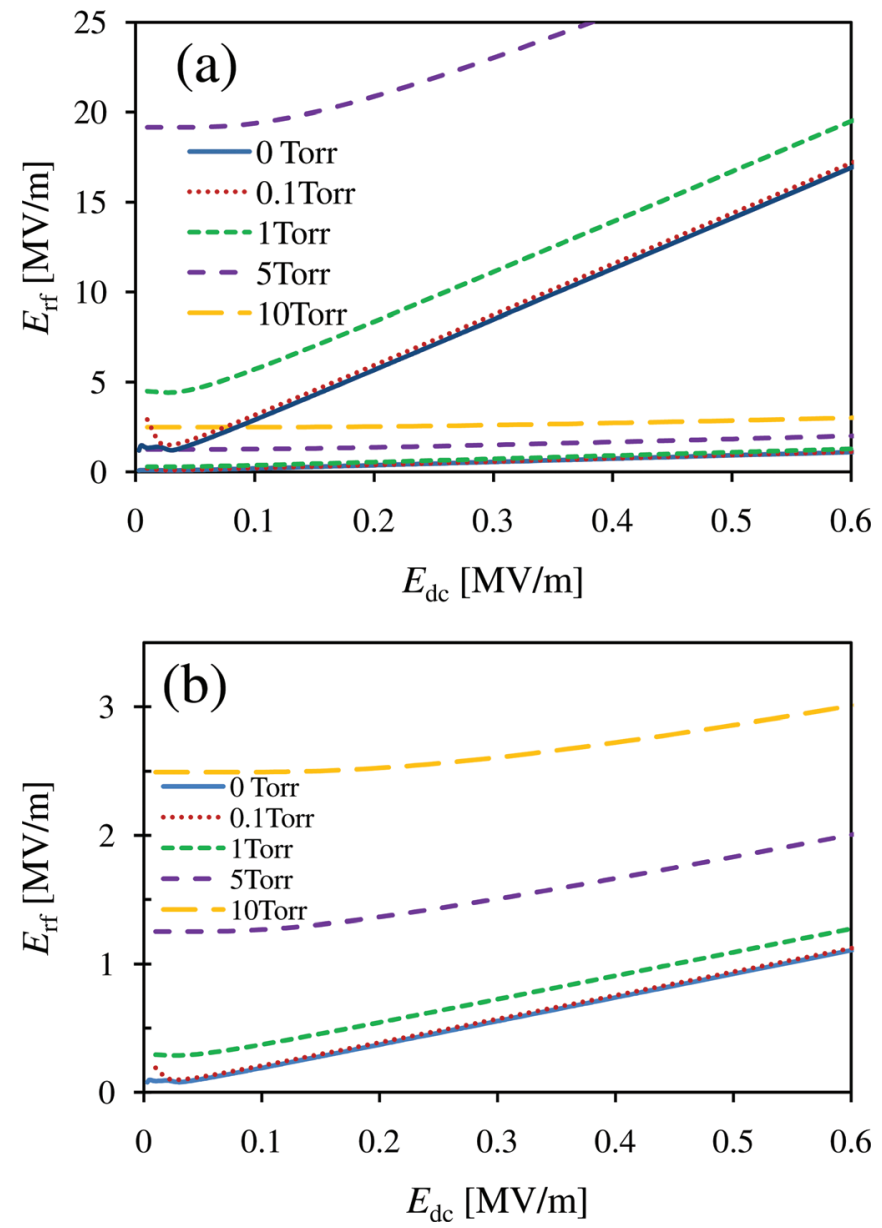

FIG. 7. (Color online) Multipactor susceptibility boundaries with gases, calculated from Eq. (23), in the $\left(E_{\mathrm{dc}}, E_{\mathrm{rf0}}\right)$ plane for $\delta_{\max 0}=3, E_{\max 0}=400 \mathrm{eV}$, $f=1 \mathrm{GHz}$, and $E_{\mathrm{om}} / E_{\max 0}=0.005$, (a) both upper and lower boundaries, (b) zoom in view of lower boundaries in (a).

electron via collisions, therefore a higher rf electric field is required to accelerate the electrons to reach the impact energy of $E_{1,2}$, clearly shown in Fig. 6. In contrast, for a given rf electric field, it is shown that multipactor saturation occurs at a lower surface charging field $E_{\mathrm{dc}}$ for higher gas pressure, since the lower boundary is shifted to the left, as shown in Fig. 6. Note that the results are similar to those obtained from analytic calculations (cf., Fig. 7 and Ref. 22). The presence of low pressure gases may promote multipactor saturation. However, if the charging dc electric field is assumed to be due to the positive charge left on the dielectric surface as a result of the ejection of the multipactor electrons by secondary emission, then we may find that the total number of multipactor electrons upon saturation $N$ is related to the charging dc electric field $E_{\mathrm{dc}}$ as $e N / 2 A \varepsilon_{0}=\sigma / 2 \varepsilon_{0} \cong E_{d c}$, where $A$ is the surface area of the dielectric and $\varepsilon_{0}$ is the free space permittivity. ${ }^{5}$ Thus, with the presence of gases, the lower surface charging field $E_{\mathrm{dc}}$ upon saturation may indicate lower saturation level of multipactor electrons $N$. At higher gas pressure, the multipactor saturation level could be so low that multipactor eventually becomes irrelevant, that is, the breakdown would be dominant by volumetric gas ionization at higher gas pressure, instead of multipactor discharge..$^{17,18,26,38}$
The upper boundary of the multipactor is even more sensitive to the presence of gases, as shown in Fig. 6(a). Due to the large value of the second crossover point $E_{2}$ in the second electron yield curve, a much higher rf power is required to overcome the electrons' collisional energy loss in order to reach the upper boundary of the multipactor susceptibility diagram.

In order to construct simple analytic solutions for the susceptibility diagrams, we once more assume that all electrons are emitted normal to the surface (i.e., $\phi=90^{\circ}$ ), with a single energy $E_{0}=(1 / 2) m v_{0}^{2}$. Hence, substituting $\phi=90^{\circ}$ into Eqs. (15) and (16), and averaging over rf phase $\theta$, the electron impact energy is found as

$$
\begin{aligned}
E(\tau) & =\frac{1}{2} m\left[\frac{1}{2 \pi} \int_{0}^{2 \pi}\left(v_{x}^{2}(\tau)+v_{y}^{2}(\tau)\right) d \theta\right] \cong \frac{m}{4 \pi} \int_{0}^{2 \pi} v_{y}^{2}(\tau) d \theta \\
& =\frac{e^{2} E_{r f}^{2}}{4 m\left(\omega^{2}+v_{t}^{2}\right)}\left[1-2 \cos (\omega \tau) e^{-v_{t} \tau}+e^{-2 v_{t} \tau}\right] .
\end{aligned}
$$

By setting $E(\tau)$ in Eq. (22) equal to $E_{1,2}$ that is obtained from Eqs. (5) and (6) after setting $\xi \cong \pi / 2$, we obtain the lower and upper boundaries of the multipactor susceptibility in the normalized form as ${ }^{22}$

$$
\bar{E}_{r f}^{2}\left(1-2 \cos (\bar{\tau}) \mathrm{e}^{-\bar{v}_{t} \bar{\tau}}+\mathrm{e}^{-2 \bar{v}_{t} \bar{\tau}}\right)=4\left(1+\bar{v}_{t}^{2}\right) \bar{E}_{1,2},
$$

where $\quad \bar{E}_{r f}=\frac{|e| E_{r f}}{\omega \sqrt{m E_{\max }}}, \quad \bar{v}_{t}=v_{t} / \omega, \quad \bar{E}_{1,2}=E_{1,2} / E_{\max o}, \quad$ and $\bar{\tau}=\omega \tau$, which is obtained by normalizing Eq. (17) as

$$
\left(1-\mathrm{e}^{-\overline{\bar{v}}_{t} \bar{\tau}}\right)\left(\sqrt{2 \bar{E}_{0}}+\frac{\bar{E}_{d c}}{\bar{v}_{t}}\right)-\bar{E}_{d c} \cdot \bar{\tau}=0,
$$

where $\bar{E}_{0}=E_{0} / E_{\max o}, \bar{E}_{d c}=\frac{|e| E_{d c}}{\omega \sqrt{m E_{\max o}}}$. It can be shown that in the limit of $v_{t} \rightarrow 0$, i.e., vacuum background, Eq. (23) recovers the known limit ${ }^{4}$

$$
\bar{E}_{r f}=\sqrt{\frac{2 \bar{E}_{1,2}}{1-\cos \left(2 \sqrt{2 \bar{E}_{o}} / \bar{E}_{d c}\right)}} .
$$

Figure 7 shows the multipactor region boundaries calculated from Eq. (23). The drastic simplification introduced to derive Eq. (23) does not qualitatively change the solution, upon comparing Fig. 7 with Fig. 6.

\section{COMBINED EFFECTS OF TANGENTIAL DC ELECTRIC FIELD AND LOW PRESSURE GAS}

We now consider the situation where both the tangential dc electric field and the low-pressure gas background are present. In this case, the conservation of momentum ${ }^{34}$ gives

$$
\begin{aligned}
m n_{e} \frac{\partial \vec{v}}{\partial t}= & -|e| n_{e}\left[\vec{E}_{r f} \sin (\omega t+\theta)+\vec{E}_{t}+\vec{E}_{d c}\right] \\
& -n_{e} m v_{m} \vec{v}-m \vec{v} G,
\end{aligned}
$$

where $\vec{v}$ is the mean electron velocity, $n_{e}$ is the electron number density, $v_{m}$ is the momentum transfer collision frequency, $G=n_{e} v_{i}$ is the electron generation rate from ionization, and $v_{i}$ is the ionization frequency. By writing 

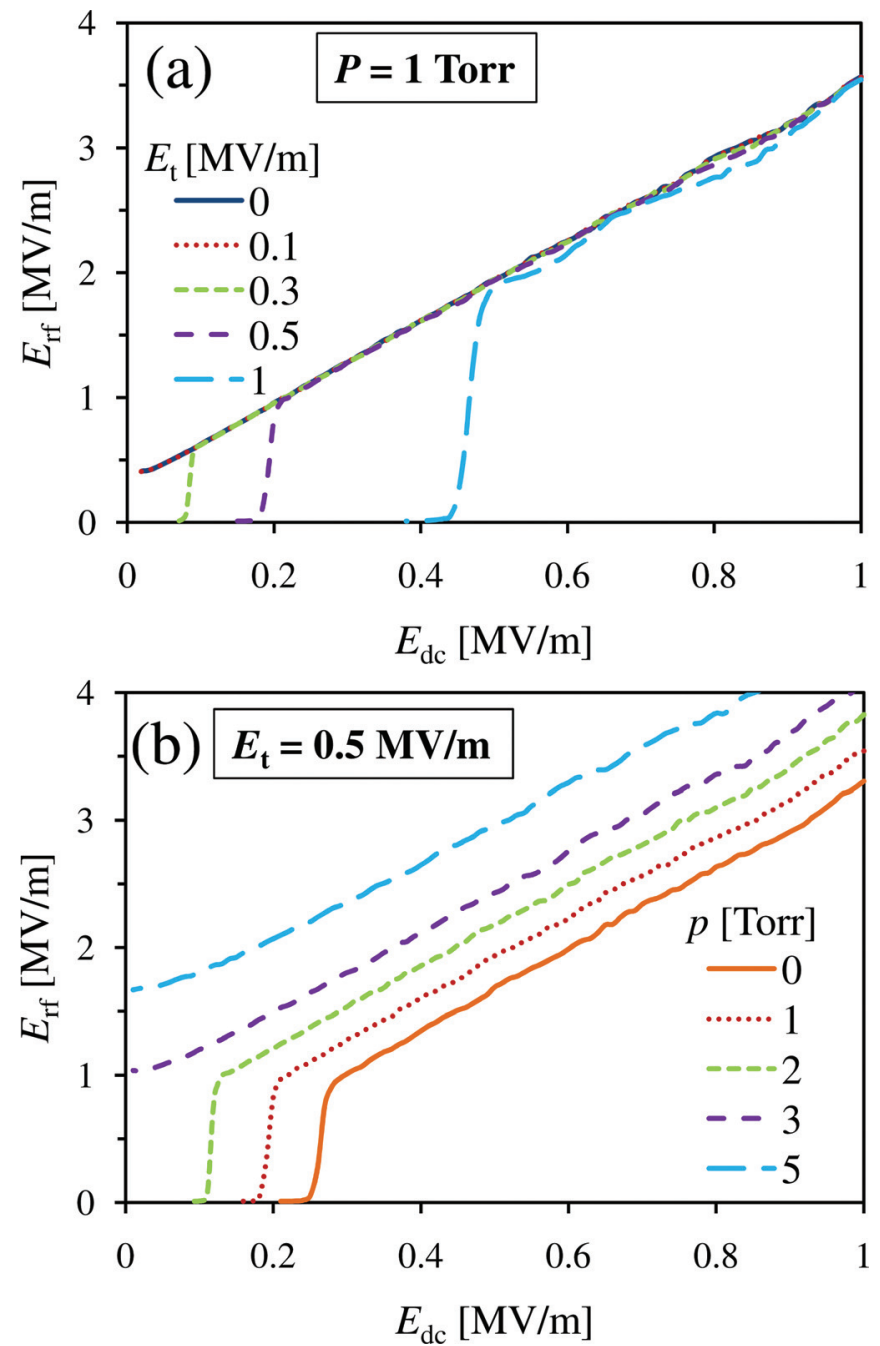

FIG. 8. (Color online) Multipactor susceptibility boundaries with both tangential dc electric field $E_{\mathrm{t}}$ and gases, from Monte Carlo simulation, in the $\left(E_{\mathrm{dc}}, E_{\mathrm{rf} 0}\right)$ plane for $\delta_{\max 0}=3, E_{\max 0}=400 \mathrm{eV}, f=1 \mathrm{GHz}$, and $E_{\mathrm{om}} / E_{\max 0}=$ 0.005 , (a) $p=1$ Torr with various $E_{\mathrm{t}}$, (b) $E_{\mathrm{t}}=0.5 \mathrm{MV} / \mathrm{m}$ with various $p$.

Eq. (26), we consider only the regime of low gas pressure, where the diffusion loss and other collisions such as excitation and recombination can be neglected. From Eq. (26) we have

$$
\begin{gathered}
\frac{\partial v_{x}}{\partial t}+v_{t} v_{x}=-\frac{|e| E_{d c}}{m} \\
\frac{\partial v_{y}}{\partial t}+v_{t} v_{y}=-\frac{|e| E_{r f} \sin (\omega t+\theta)}{m}-\frac{|e| E_{t}}{m}
\end{gathered}
$$

where $v_{t}=v_{i}+v_{m}$. Solving Eqs. (27) and (28), we have

$$
\begin{aligned}
& v_{x}=-\frac{|e| E_{d c}}{m v_{t}}+\mathrm{e}^{-v_{t} t}\left(v_{0} \sin \phi+\frac{|e| E_{d c}}{m v_{t}}\right), \\
v_{y}= & \mathrm{e}^{-v_{t} t}\left(v_{0} \cos \phi+\frac{|e| E_{t}}{m v_{t}}\right)+\frac{|e| E_{r f} \mathrm{e}^{-v_{t} t}}{m\left(\omega^{2}+v_{t}^{2}\right)} \\
& \times\left(v_{t} \sin \theta-\omega \cos \theta\right)-\frac{|e| E_{r f}}{m\left(\omega^{2}+v_{t}^{2}\right)} \\
& \times\left[v_{t} \sin (\omega t+\theta)-\omega \cos (\omega t+\theta)\right]-\frac{|e|}{m} \frac{E_{t}}{v_{t}},
\end{aligned}
$$

where $v_{0}$ is the initial emission velocity. The transit time is still given by Eq. (17). Similar to Sec. III, we assume $v_{t} / p \approx 10^{10} \mathrm{Torr}^{-1} s^{-1}$ in our calculations.

For the MC simulation, we apply the same approach as in Secs. II and III to obtain the multipactor region boundaries. Figure 8(a) shows the boundaries of multipactor susceptibility for $p=1$ Torr, with various tangential dc electric fields. Figure 8(b) shows the boundary for $E_{\mathrm{t}}=0.5 \mathrm{MV} / \mathrm{m}$, but with various gas pressures. As seen earlier, the upper boundaries for multipactor are not sensitive to the tangential dc electric field. With the presence of gases, a much larger rf power is required to reach the upper boundary of the multipactor susceptibility. Thus we focus on the lower boundaries of the multipactor region. Figure 8(a) shows that an increase in the tangential dc field $E_{\mathrm{t}}$ will lower the lower boundary of the multipactor susceptibility for the rf electric field. Figure 8(b) shows that an increase in pressure will raise the lower boundary of the multipactor regions for the rf electric field. In order to make notable lowering in the lower boundary, a larger tangential dc field has to be applied for higher gas pressures.

After averaging over the rf phase $\theta$ and setting the average impact energy equal to $E_{1,2}$ that is obtained from Eqs.
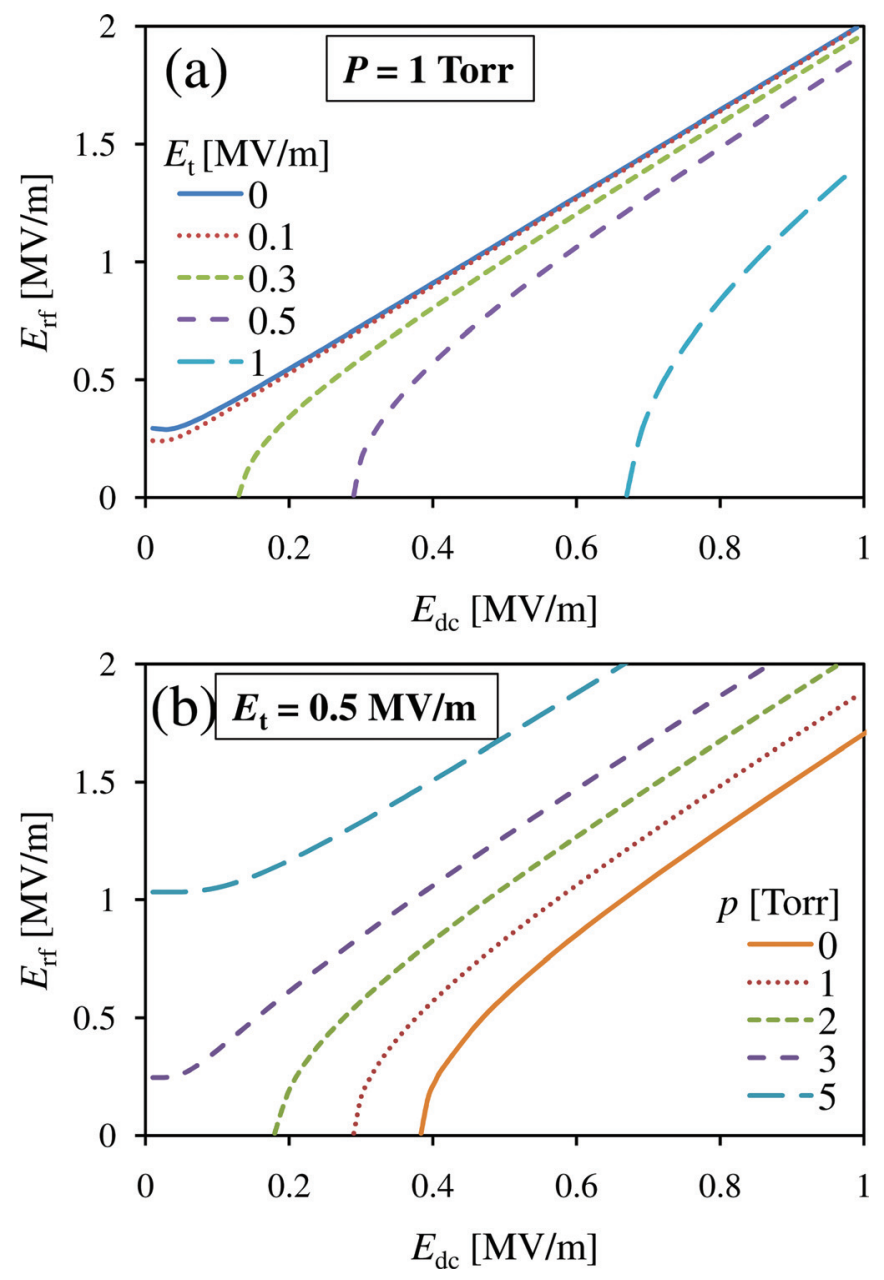

FIG. 9. (Color online) Multipactor susceptibility boundaries with both tangential dc electric field $E_{\mathrm{t}}$ and gases, calculated from Eq. (31), in the ( $E_{\mathrm{dc}}$, $\left.E_{\mathrm{rf0}}\right)$ plane for $\delta_{\max 0}=3, E_{\max 0}=400 \mathrm{eV}, f=1 \mathrm{GHz}$, and $E_{\mathrm{om}} / E_{\max 0}=$ 0.005 , (a) $p=1$ Torr with various $E_{\mathrm{t}}$, (b) $E_{\mathrm{t}}=0.5 \mathrm{MV} / \mathrm{m}$ with various $p$. 
(5) and (6) after setting $\xi \cong \pi / 2$, we obtain the analytical solutions for the lower and upper boundaries of the multipactor susceptibility in the normalized form

$$
\begin{aligned}
& \bar{E}_{r f}^{2}\left(1-2 \cos (\bar{\tau}) \mathrm{e}^{-\bar{v}_{t} \bar{\tau}}+\mathrm{e}^{-2 \bar{v}_{t} \bar{\tau}}\right)+2 \bar{E}_{t}^{2}\left(1-\mathrm{e}^{-\bar{v}_{t} \bar{\tau}}\right)^{2}\left(1+\bar{v}_{t}^{2}\right) / \bar{v}_{t}^{2} \\
& \quad=4\left(1+\bar{v}_{t}^{2}\right) \bar{E}_{1,2},
\end{aligned}
$$

where all the terms are defined earlier in Secs. II and III.

Figure 9 shows the multipactor region boundaries calculated from Eq. (31). The drastic simplification introduced to derive Eq. (31) does not qualitatively change the solution, upon comparing Fig. 9 with Fig. 8. Thus, Eq. (31) may be used for a rough evaluation of multipactor susceptibility under practical conditions where both tangential dc electric field and low pressure gases are present.

\section{CONCLUDING REMARKS}

In this paper, we study the combined effects of a tangential dc electric field and of an external gas at a low pressure on the multipactor initiation. The presence of a tangential dc electric field lowers the magnitude of rf electric field threshold to initiate multipactor, therefore potentially providing a more robust mechanism to block HPM. For a reasonably high rf electric field, (above the threshold for multipactor initiation under vacuum), the presence of low pressure gases may promote multipactor saturation, but at a lower saturation level. For the case in which both tangential dc electric field and external gases are included, the multipactor susceptibility is obtained. It is found that a larger tangential dc field needs to be applied for higher gas pressures in order to make a notable reduction for the threshold rf electric field corresponding to the lower boundary of the susceptibility curve.

This paper may be extended in several directions, some of fundamental interest. The natural extension concerns the inter-relation between the Paschen curve, dc dielectric flashover, and the scaling laws for rf window breakdown. This critical examination may lead to an interesting generalization of the classical Paschen curve to include the effect of side walls during gas breakdown. The theory developed in this paper is to be compared with an ongoing proof-of-principle experiment at the University of Michigan.

\section{ACKNOWLEDGMENTS}

The authors would like to thank Dr. John Luginsland, Dr. David French, and Dr. Brad Hoff for helpful discussions. This work was supported by an AFOSR grant on the Basic Physics of Distributed Plasma Discharges, L-3 Communications Electron Device Division, and Northrop-Grumman Corporation. One of us (PZ) gratefully acknowledges a fellowship from the University of Michigan Institute for Plasma Science and Engineering.
${ }^{1}$ J. R. M. Vaughan, IEEE Trans. Electron Devices 35, 1172 (1988).

${ }^{2}$ J. R. M. Vaughan, IEEE Trans. Electron Devices 8, 302 (1961).

${ }^{3}$ D. H. Preist and R. C. Talcott, IRE Trans. Electron Devices 8, 243 (1961).

${ }^{4}$ R. A. Kishek and Y. Y. Lau, Phys. Rev. Lett. 80, 193 (1998).

${ }^{5}$ L. K. Ang, Y. Y. Lau, R. A. Kishek, and R. M. Gilgenbach, IEEE Trans. Plasma Sci. 26, 290 (1998).

${ }^{6}$ R. A. Kishek, Y. Y. Lau, L. K. Ang, A. Valfells, and R. M. Gilgenbach, Phys. Plasmas 5, 2120 (1998).

${ }^{7}$ A. Neuber, J. Dickens, D. Hemmert, H. Krompholz, L. Hatfield, and M. Kristiansen, IEEE Trans. Plasma Sci. 26, 296 (1998).

${ }^{8}$ J. Foster, H. Krompholz, and A. Neuber, Phys. Plasmas 18, 013502 (2011).

${ }^{9}$ A. A. Neuber, L. Laurent, Y. Y. Lau, and H. Krompholz, in High Power Microwave Sources and Technologies, edited by R. J. Barker and E. Schamiloglu (IEEE, Piscataway, NJ, 2001), Chap. 10, p. 325.

${ }^{10}$ A. Sazontov, V. Semenov, and M. Buyanova, N. Vdovicheva, D. Anderson, M. Lisak, J. Puech, and L. Lapierre, Phys. Plasmas 12, 093501 (2005).

${ }^{11}$ U. Jordan, D. S. Dorozhkina, V. E. Semenov, T. Olsson, D. Anderson, M. Lisak, J. Puech, I. M. Nefedov, and I. A. Shereshevskii, IEEE Trans. Plasma Sci. 35, 542 (2007).

${ }^{12}$ J. G. Power, W. Gai, S. H. Gold, A. K. Kinkead, R. Konecny, C. Jing, W. Liu, and Z. Yusof, Phys. Rev. Lett. 92, 164801 (2004).

${ }^{13}$ L. Wu and L. K. Ang, Phys. Plasmas 14, 013105 (2007).

${ }^{14}$ B. W. Hoff, P. J. Mardahl, R. M. Gilgenbach, M. D. Haworth, D. M. French, Y. Y. Lau, and M. Franzi, Rev. Sci. Instrum. 80, 094702 (2009).

${ }^{15}$ R. Woo, Proc. IEEE 57, 254 (1969); A. Gilmour, Microwave Tubes (Artech House, Norwood, MA, 1986), Chap. 15.

${ }^{16}$ M. Rubin, Radar-The Electronic Eye (Chemical Publishing Co. Inc., New York, 1963), Chap. 9.

${ }^{17}$ H. C. Kim and J. P. Verboncoeur, Phys. Plasmas 13, 123506 (2006).

${ }^{18}$ H. C. Kim, J. P. Verboncoeur, and Y. Y. Lau, IEEE Trans. Electr. Insul. 14, 766 (2007).

${ }^{19}$ A. Valfells, J. P. Verboncoeur, and Y. Y. Lau, IEEE Trans. Plasma Sci. 28, 529 (2000); C. Chang, G. Z. Liu, C. X. Tang, L. X. Yan, Phys. Plasmas 16, 053506 (2009); A. Neuber, D. Hemmert, H. Krompholz, L. L. Hatfield, and M. Kristiansen, J. Appl. Phys. 86, 1724 (1999).

${ }^{20}$ A. Valfells, L. K. Ang, Y. Y. Lau, and R. M. Gilgenbach, Phys. Plasmas 7, 093508 (2000).

${ }^{21}$ A. G. Sazontov and V. E. Nevchaev, Phys. Plasmas 17, 033509 (2010).

${ }^{22}$ C. Chang, G. Liu, C. Tang, C. Chen, S. Qiu, J. Fang, and Q. Hou, Phys. Plasmas 15, 093508 (2008).

${ }^{23}$ O. A. Ivanov, M. A. Lobaev, V. A. Isaev, and A. L. Vikharev, Phys. Rev. ST Accel. Beams 13, 022004 (2010).

${ }^{24}$ S. K. Nam and J. P. Verboncoeur, Appl. Phys. Lett. 92, 231502 (2008).

${ }^{25}$ S. K. Nam, C. Lim, and J. P. Verboncoeur, Phys. Plasmas 16, 023501 (2009).

${ }^{26}$ Y. Y. Lau, J. P. Verboncoeur, and H. C. Kim, Appl. Phys. Lett. 89, 261501 (2006).

${ }^{27}$ H. C. Miller, in High Voltage Vacuum Insulation, edited by R. V. Latham (Academic, London, 1995), Chap. 8, p.310.

${ }^{28}$ H. C. Miller, IEEE Trans. Electr. Insul. 24, 765 (1989).

${ }^{29}$ K. D. Bergeron, J. Appl. Phys. 48, 3073 (1977).

${ }^{30}$ R. A. Anderson and J. P. Brainard, J. Appl. Phys. 51, 1414 (1980).

${ }^{31}$ M. E. Cuneo, IEEE Trans. Dielectr. Electr. Insul. 6, 469 (1999).

${ }^{32}$ N. M. Jordan, Y. Y. Lau, D. M. French, R. M. Gilgenbach, and P. Pengvanich, J. Appl. Phys. 102, 033301 (2007).

${ }^{33}$ J. R. M. Vaughan, IEEE Trans. Electron Devices 36, 1963 (1989).

${ }^{34}$ M. A. Lieberman and A. J. Lichtenberg, Principles of Plasma Discharges and Materials Processing, 2nd ed. (John Wiley \& Sons, Hoboken,New Jersey, 2005), p. 31.

${ }^{35}$ Y. Nakamura and M. Kurachi, J. Phys.D 21, 718 (1988).

${ }^{36}$ M. Hayashi and S. Ushiroda, J. Chem. Phys. 78(5), 2621 (1983).

${ }^{37}$ D. Rapp and P. Englander-Golden, J. Chem. Phys. 43(5), 1464 (1965).

${ }^{38}$ J. P. Verboncoeur, H. C. Kim, Y. Chen, and Y. Y. Lau, "Modeling RF window breakdown: From vacuum multipactor to volumetric ionization discharge," 27th IEEE International Power Modulator Symposium, Washington D.C., USA, 2006. 